December 2005

\title{
Global properties of charged dilatonic Gauss-Bonnet black holes
}

\author{
M. Melis ${ }^{\dagger}$ and S. Mignemi ${ }^{\ddagger *}$ \\ Dipartimento di Matematica, Università di Cagliari \\ viale Merello 92, 09123 Cagliari, Italy
}

\begin{abstract}
We study the phase space of the spherically symmetric solutions of Einstein-Maxwell-Gauss-Bonnet system nonminimally coupled to a scalar field and prove the existence of solutions with unusual asymptotics in addition to asymptotically flat ones. We also find new dyonic solutions of dilatonic Einstein-Maxwell theory.
\end{abstract}

P.A.C.S. Numbers: 97.60.Lf 11.25.Mj

\footnotetext{
$\dagger$ e-mail: maurizio.melis@ca.infn.it

$\ddagger$ e-mail: smignemi@unica.it

* and INFN, Sezione di Cagliari
} 


\section{Introduction}

In a recent paper [1] we have studied the global properties of the spherically symmetric solutions of Einstein-Gauss-Bonnet (GB) gravity nonminimally coupled to a scalar field. We refer to that paper for a full discussion of the relevance of GB gravity for physics and for a list of references. We only recall its role in the low-energy field theory expansion of string theory [2].

Our investigation showed that in four dimensions the only regular black hole solutions of the system are asymptotically flat. It is interesting to check if this property holds also for charged black holes. In fact, this is not the case. Already in absence of GB coupling some degenerate charged solutions of dilatonic gravity with unusual asymptotic behaviour are known [3]. Actually, analytical solution to dilatonic gravity with nontrivial Maxwell field are well known in absence of GB coupling [4,5]. If only magnetic or electric charges are present, the solutions display only one horizon and are analogous to Schwarzschild black holes. If instead both charges are nonvanishing, they possess an inner and an outer horizon and the causal structure is similar to that of the Reissner-Nordström solution. Asymptotically, they are flat except in the degenerate cases cited above [3].

In the case of dilatonic Einstein-GB models, magnetically charged solutions cannot be obtained in analytical form, but have been studied perturbatively [6] and numerically $[7,8]$. Only asymptotically flat solutions have been investigated in this context. The existence of solutions with different asymptotics can however be decided through the study of the global properties of the phase space associated with the dynamical system, as has been shown in the literature in analogous situations [9]. It must be noticed that the search for solution is made easier in the Einstein case by the existence of a duality between electrically and magnetically charged solutions, that is no longer available in the presence of GB corrections.

Also interesting is the study of the emergence of branch singularities in the metrics. This is a common feature of GB models [10,7], and can give rise to naked singularities. This topic can be investigated by means of an expansion of the fields near the outer horizon [11].

In this paper, we study the dilatonic Einstein-GB system coupled to Maxwell field, and classify the solutions by their asymptotic behaviour. We find that their behaviour is the same as in the dilatonic Einstein case, except for some non-asymptotically flat purely magnetic solutions, which do not have analogues if GB terms are included. In the course of the investigation, we also find new dyonic solutions of dilatonic Einstein gravity with unusual asymptotics. We also study the near-horizon expansion of the metric functions, finding the conditions for the absence of naked branch singularities and show the existence of solutions with multiple horizons.

\section{The dynamical system}

In this section, using the methods of ref. [1], we derive the dynamical system associated to spherically symmetric solutions of the field equations of dilatonic Einstein-GB-Maxwell theory. For further technical details we refer to that paper. 
The four-dimensional action of four-dimensional Einstein-GB gravity coupled to electromagnetism is

$$
I=\frac{1}{16 \pi G} \int \sqrt{-g} d^{4} x\left[\mathcal{R}-2(\partial \phi)^{2}+\alpha e^{-2 \phi}\left(\mathcal{S}-F^{2}\right)\right]
$$

where $\mathcal{S}=\mathcal{R}^{\mu \nu \rho \sigma} \mathcal{R}_{\mu \nu \rho \sigma}-4 \mathcal{R}^{\mu \nu} \mathcal{R}_{\mu \nu}+\mathcal{R}^{2}$ is the GB term, $F$ is the Maxwell field, $\phi$ is a scalar field and $\alpha$ is a constant with dimension (length) ${ }^{2}$. This action also emerges in the low-energy expansion of string theory [2], and in this case the constant $\alpha$ is proportional to the slope parameter. Note, however, that in string theory also an axion field may couple to the gravitational and Maxwell fields, and this may change notably the properties of the solutions [12].

For the metric we adopt the spherically symmetric ansatz

$$
d s^{2}=-\Delta d t^{2}+\Delta^{-1} d r^{2}+R^{2} d \Omega^{2}
$$

where $\Delta$ and $R$ are functions of $r$. In these coordinates the generalized Maxwell equations

$$
\nabla^{\mu}\left(e^{-2 \phi} F_{\mu \nu}\right)=0
$$

admit the spherically symmetric solution

$$
F=q_{e} \frac{e^{2 \phi}}{R^{2}} d t \wedge d r+q_{m} \sin \theta d \theta \wedge d \phi
$$

where $q_{e}$ and $q_{m}$ are the electric and the magnetic charge, respectively.

The field equations for the gravitational and scalar degrees of freedom following from (1) and (4) are more conveniently written in terms of new variables such that the metric and the scalar take the form [1]

$$
d s^{2}=-e^{\eta+\chi} d t^{2}+e^{4 \zeta-\eta-\chi} d \xi^{2}+e^{2 \zeta-\eta-\chi} d \Omega^{2}, \quad 2 \phi=\eta-\chi
$$

where $\zeta, \eta, \chi$ and $\lambda$ are functions of $\xi$. They read

$$
\begin{aligned}
& \zeta^{\prime \prime}-\alpha\left[\left(\eta^{\prime 2}-\chi^{\prime 2}\right)\left(\eta^{\prime}+\chi^{\prime}-2 \zeta^{\prime}\right) e^{2 \chi-4 \zeta}\right]^{\prime}=e^{2 \zeta}+\alpha\left(\eta^{2}-\chi^{\prime 2}\right)\left[2 e^{2 \chi-2 \zeta}-\left(\eta^{\prime}+\chi^{\prime}-2 \zeta^{\prime}\right)^{2} e^{2 \chi-4 \zeta}\right] \\
& \eta^{\prime \prime}+\alpha\left[4 \eta^{\prime} e^{2 \chi-2 \zeta}-\left(\eta^{\prime}+\chi^{\prime}-2 \zeta^{\prime}\right)\left(2 \eta^{\prime 2}-\chi^{\prime 2}+\eta^{\prime} \chi^{\prime}-2 \eta^{\prime} \zeta^{\prime}\right) e^{2 \chi-4 \zeta}\right]^{\prime}=2 \alpha q_{e}^{2} e^{2 \eta} \\
& \chi^{\prime \prime}-\alpha\left[4 \chi^{\prime} e^{2 \chi-2 \zeta}+\left(\eta^{\prime}+\chi^{\prime}-2 \zeta^{\prime}\right)\left(\eta^{\prime 2}-2 \chi^{\prime 2}-\eta^{\prime} \chi^{\prime}+2 \eta^{\prime} \zeta^{\prime}\right) e^{2 \chi-4 \zeta}\right]^{\prime}= \\
& 2 \alpha q_{m}^{2} e^{2 \chi}+\alpha\left(\eta^{\prime 2}-\chi^{\prime 2}\right)\left[4 e^{2 \chi-2 \zeta}-\left(\eta^{\prime}+\chi^{\prime}-2 \zeta^{\prime}\right)^{2} e^{2 \chi-4 \zeta}\right] \\
& 2 \zeta^{\prime 2}-\eta^{\prime 2}-\chi^{\prime 2}-\alpha\left(\eta^{\prime 2}-\chi^{\prime 2}\right)\left[4 e^{2 \chi-2 \zeta}-3\left(\eta^{\prime}+\chi^{\prime}-2 \zeta^{\prime}\right)^{2} e^{2 \chi-4 \zeta}\right]=2 e^{2 \zeta}-2 \alpha q_{m}^{2} e^{2 \chi}-2 \alpha q_{e}^{2} e^{2 \eta}
\end{aligned}
$$

It is interesting to notice that in spite of the presence of the higher-derivative GB term, the field equations are second order.

We can then write (6) in the form of a first order dynamical system by defining new variables

$$
X=\zeta^{\prime}, \quad Y=\eta^{\prime}, \quad W=\chi^{\prime}, \quad Z=e^{\zeta}, \quad U=\sqrt{\alpha} e^{\eta}, \quad V=\sqrt{\alpha} e^{\chi}
$$


which satisfy the differential equations

$$
Z^{\prime}=X Z, \quad U^{\prime}=Y U, \quad V^{\prime}=W V
$$

In terms of these variables, the field equations become

$$
\begin{gathered}
X^{\prime}-\left\{\left(Y^{2}-W^{2}\right)(Y+W-2 X) \frac{V^{2}}{Z^{4}}\right\}^{\prime}=Z^{2}+\left(Y^{2}-W^{2}\right)\left[2 Z^{2}-(Y+W-2 X)^{2}\right] \frac{V^{2}}{Z^{4}}, \\
Y^{\prime}+\left\{\left[4 Y Z^{2}-(Y+W-2 X)\left(2 Y^{2}-W^{2}+Y W-2 X Y\right)\right] \frac{V^{2}}{Z^{4}}\right\}^{\prime}=2 q_{e}^{2} U^{2}, \\
W^{\prime}-\left\{\left[4 W Z^{2}+(Y+W-2 X)\left(Y^{2}-2 W^{2}-Y W+2 X W\right)\right] \frac{V^{2}}{Z^{4}}\right\}^{\prime}= \\
2 q_{m}^{2} V^{2}+\left(Y^{2}-W^{2}\right)\left[4 Z^{2}-(Y+W-2 X)^{2}\right] \frac{V^{2}}{Z^{4}},
\end{gathered}
$$

subject to the constraint

$$
E=2 X^{2}-Y^{2}-W^{2}-2 Z^{2}+2 q_{m}^{2} V^{2}+2 q_{e}^{2} U^{2}-\left(Y^{2}-W^{2}\right)\left[4 Z^{2}-3(Y+W-2 X)^{2}\right] \frac{V^{2}}{Z^{4}}=0 .
$$

In general, the dynamical system is six-dimensional, but for purely magnetic solutions $\left(q_{e}=0\right)$, the variable $U$ is ignorable and does not appear in the system, while (10) yields the first integral

$$
Y+\left[4 Y Z^{2}-(Y+W-2 X)\left(2 Y^{2}-W^{2}+Y W-2 X Y\right)\right] \frac{V^{2}}{Z^{4}}=a .
$$

In this case the dynamical system is effectively four-dimensional. We shall nevertheless work also in this case with the full six-dimensional phase space, because this facilitates the discussion of the critical points. For the same reason, we shall not use the constraint (12) to eliminate $V^{2} / Z^{4}$ and reduce by one the dimensionality of the phase space.

The system can be put in canonical form solving (9)-(11) for the first derivatives, as

$$
X^{\prime}=F(X, Y, W, Z, U, V), \quad Y^{\prime}=G(X, Y, W, Z, U, V), \quad W^{\prime}=H(X, Y, W, Z, U, V) .
$$

The expressions so obtained are awkward and shall not be reported here.

\section{Charged solutions of dilatonic Einstein gravity}

In the following, the knowledge of the exact solutions in absence of the GB term will prove useful. These were first discussed in [4], and later some degenerate cases with nontrivial asymptotics were found in [2] for the magnetic monopole. We shall generalize these solutions to the dyonic case. An important property of the dilatonic Einstein-Maxwell theory is the presence of a duality invariance for the interchange of $q_{e}$ and $q_{m}$, with $\phi \rightarrow-\phi$.

In absence of Gauss-Bonnet contributions, the field equations (6) reduce to

$$
\zeta^{\prime \prime}=e^{2 \zeta}, \quad \eta^{\prime \prime}=2 q_{e}^{2} e^{2 \eta}, \quad \chi^{\prime \prime}=2 q_{m}^{2} e^{2 \chi},
$$


subject to the constraint

$$
2 \zeta^{\prime 2}-\eta^{\prime 2}-\chi^{\prime 2}-2 e^{2 \zeta}+2 q_{m}^{2} e^{2 \chi}+2 q_{e}^{2} e^{2 \eta}=0
$$

where we have absorbed $\alpha$ into the definition of the charges. In these variables, the duality invariance corresponds to the interchange of $\eta$ and $\chi$. The integration of (15) yields

$$
e^{\zeta}=\frac{2 a e^{a \xi}}{1-e^{2 a \xi}}, \quad e^{\eta}=\frac{\sqrt{2}}{\left|q_{e}\right|} \frac{b e^{b\left(\xi-\xi_{1}\right)}}{1-e^{2 b\left(\xi-\xi_{1}\right)}}, \quad e^{\chi}=\frac{\sqrt{2}}{\left|q_{m}\right|} \frac{c e^{c\left(\xi-\xi_{2}\right)}}{1-e^{2 c\left(\xi-\xi_{2}\right)}},
$$

where $a, b, c, \xi_{1}$ and $\xi_{2}$ are integration constants and the origin of $\xi$ has been fixed arbitrarily. Substituting in the constraint (16), one gets $2 a^{2}-b^{2}-c^{2}=0$. If one also requires that the solution has a regular horizon, one must impose that $R^{2}=e^{2 \zeta-\eta-\chi}$ is regular at the horizon, $\xi \rightarrow-\infty$, which implies $2 a-b-c=0$. Combined with the previous constraint, this yields $a=b=c$. Substituting these results in (5) and defining a new coordinate $r=\int e^{2 \zeta} d \xi=2 a /\left(1-e^{2 a \xi}\right)$, one obtains the solution

$$
\begin{aligned}
d s^{2} & =-\frac{r\left(r-r_{h}\right)}{\left(r+r_{e}\right)\left(r+r_{m}\right)} d t^{2}+\frac{\left(r+r_{e}\right)\left(r+r_{m}\right)}{r\left(r-r_{h}\right)} d r^{2}+\left(r+r_{e}\right)\left(r+r_{m}\right) d \Omega^{2}, \\
e^{-2 \phi} & =\frac{\left(r+r_{e}\right)}{\left(r+r_{m}\right)},
\end{aligned}
$$

where $r_{h}=2 a$ and $r_{e}$ and $r_{m}$ are functions of $a, \xi_{1}$ and $\xi_{2}$. This solution was first discussed in ref. [4]. The mass $M$ and the charges of the black hole are related to the parameters of the solution by

$$
2 M=r_{h}+r_{e}+r_{m}, \quad q_{e}^{2}=\frac{r_{e}\left(r_{h}+r_{e}\right)}{2}, \quad q_{m}^{2}=\frac{r_{m}\left(r_{h}+r_{m}\right)}{2} .
$$

The metric (18) has two horizons at $r=r_{h}$ and $r=0$, a singularity at $r=\max \left(-r_{e},-r_{m}\right)$, and is asymptotically flat. The special case when $q_{e}=0$ was also derived in [5] and corresponds to $r_{e} \rightarrow 0$.

Some degenerate solutions with unusual asymptotics arise when $\xi_{1}$ or $\xi_{2}$ vanish. For example, for $\xi_{2}=0$, one has

$$
d s^{2}=-\frac{r\left(r-r_{h}\right)}{r+r_{e}} d t^{2}+\frac{r+r_{e}}{r\left(r-r_{h}\right)} d r^{2}+\left(r+r_{e}\right) d \Omega^{2}, \quad e^{-2 \phi}=\frac{r+r_{e}}{2 q_{m}^{2}} .
$$

For $\xi_{1}=0$, the metric has the same form, with $r_{e} \rightarrow r_{m}$, while the scalar becomes $e^{-2 \phi}=2 q_{e}^{2} /\left(r+r_{m}\right)$. In different coordinates the metric (20) can be also written

$$
d s^{2}=-\frac{\left(R^{2}-r_{e}\right)\left(R^{2}-r_{0}\right)}{R^{2}} d t^{2}+\frac{R^{4}}{\left(R^{2}-r_{e}\right)\left(R^{2}-r_{0}\right)} d R^{2}+R^{2} d \Omega^{2},
$$

with $e^{-2 \phi}=R^{2} / 2 q_{m}^{2}$, and $r_{0}=r_{h}+r_{e}$. The solution (20) generalizes that found in [3] for the purely magnetic case, which is recovered in the limit $r_{e} \rightarrow 0$. In the general case, the metric (20) displays an outer horizon at $r=r_{h}$, an inner horizon at $r=0$, and a singularity at $r=-r_{e}$. Its asymptotic behaviour is intermediate between Schwarzschild and anti-de Sitter and its causal structure is similar to that of the Reissner-Nordström metric.

For $\xi_{1}=\xi_{2}=0$, one obtains a previously unknown solution, of the form

$$
d s^{2}=-\frac{r\left(r-r_{h}\right)}{2 q_{e} q_{m}} d t^{2}+\frac{2 q_{e} q_{m}}{r\left(r-r_{h}\right)} d r^{2}+2 q_{e} q_{m} d \Omega^{2}, \quad e^{-2 \phi}=\frac{q_{e}}{q_{m}} .
$$


This metric is the direct product of two-dimensional anti-de Sitter spacetime with a two-dimensional sphere and is therefore of the Bertotti-Robinson form. It has a horizons at $r=0$ and $r=r_{h}$, but no singularity, and both nonvanishing electric and magnetic charge.

In all cases, extremal black holes are obtained in the limit $a=0$, corresponding to $r_{h}=0$, or, in terms of the physical parameters, $2 M^{2}=\left(q_{e} \pm q_{m}\right)^{2}$.

Finally, we notice that in terms of the variables of the dynamical system the solution (17) reads

$$
\begin{gathered}
X=a \operatorname{coth}(a \xi), \quad Y=a \operatorname{coth}\left[a\left(\xi-\xi_{1}\right)\right], \quad W=a \operatorname{coth}\left[a\left(\xi-\xi_{2}\right)\right], \\
Z=\frac{a}{\sinh (a \xi)}, \quad U=\frac{a}{\sqrt{2}\left|q_{e}\right| \sinh \left[a\left(\xi-\xi_{1}\right)\right]}, \quad V=\frac{a}{\sqrt{2}\left|q_{m}\right| \sinh \left[a\left(\xi-\xi_{2}\right)\right]},
\end{gathered}
$$

with the horizon at $\xi \rightarrow-\infty$, and spatial infinity at $\xi=0$. From the explicit knowledge of the solutions or by standard methods, it is then easy to construct the phase space portrait. Phase space is invariant under $Z \rightarrow-Z, U \rightarrow-U$ or $V \rightarrow-V$, thus we shall limit our study to positive values of these variables.

The physical trajectories lie on the five-dimensional hyperplane $E=0$. The critical point at finite distance lie on the surface $Z_{0}=U_{0}=V_{0}=P_{0}=0$, where

$$
P^{2} \equiv 2 X^{2}-Y^{2}-W^{2}
$$

but only points with $X_{0}=Y_{0}=W_{0}$ correspond to regular horizons. The eigenvalues of the linearized equations around the critical points are $0(3), X_{0}, Y_{0}, W_{0}$.

The phase space at infinity can be studied defining new variables

$$
t=\frac{1}{X}, \quad y=\frac{Y}{X}, \quad w=\frac{W}{X}, \quad z=\frac{Z}{X}, \quad u=\frac{U}{X}, \quad v=\frac{V}{X} .
$$

The critical points at infinity are found at $t_{0}=0$ and

a) $u_{0}=v_{0}=z_{0}=0, y=y_{0}, w=w_{0}$, with $y_{0}^{2}+w_{0}^{2}=2$. These are endpoints of trajectories lying on the surface at infinity.

b) $u_{0}=v_{0}=0, z_{0}=1, y_{0}=w_{0}=0$. These are the endpoints of the asymptotically flat solutions (18).

c) $u_{0}=0, v_{0}=1 / \sqrt{2 q_{m}^{2}}, z_{0}=1, y_{0}=0, w_{0}=1$. These are the endpoints of the solutions (20).

d) $v_{0}=0, u_{0}=1 / \sqrt{2 q_{e}^{2}}, z_{0}=1, y_{0}=1, w_{0}=0$. These are the endpoints of the dual solutions to (20).

e) $u_{0}=1 / \sqrt{2 q_{e}^{2}}, v_{0}=1 / \sqrt{2 q_{m}^{2}}, z_{0}=1, y_{0}=w_{0}=1$. These are the endpoints of the solutions (22).

The eigenvalues of the linearized equations are
a) $0(3), 1, y_{0}, w_{0}$.
b) $-1(5),-2$.
c) $1,-1(3),-2(2)$.
d) $1,-1(3),-2(2)$.
e) $1(2),-1,-2(3)$. 
In absence of electric charge, the system reduces to a four-dimensional system with $U=Y=0$, and similarly for $q_{m}=0$, it reduces to a system with $V=W=0$.

The phase space portrait is the following: regular solutions start at $Z_{0}=U_{0}=V_{0}=0, \quad X_{0}=Y_{0}=$ $W_{0}=a$, for some value of the parameter $a$. At infinity, a three-dimensional family of trajectories is attracted by points b) and a two-dimensional bunch by points c) and d). Finally, a one-dimensional bunch ends at the critical point e).

\section{Phase space of the Einstein-GB system}

We discuss now the phase space associated with the Einstein-GB system (8), (14). Since $E$ in (12) is a first integral of the dynamical system, all the trajectories are confined to surfaces of constant $E$, but only those with vanishing $E$ are physical. We shall therefore consider only the critical points lying on the hypersurface $E=0$. The discussion of the phase space is complicated by the presence of a singularity in the field equations at $Z=0$. Since some of the critical points of the dynamical system lie on this hypersurface, one must be careful in taking the limits when approaching these points. In particular, when $Z \rightarrow 0$, also $V \rightarrow 0$. Although the duality invariance is no longer valid, the phase space is still invariant under $Z \rightarrow-Z$, $U \rightarrow-U$ or $V \rightarrow-V$, as in the pure Einstein case. We can therefore consider only positive values of $Z, U$ and $V$.

The critical points at finite distance are defined as the points were the r.h.s. of eqs. (8) and (14) vanishes, and in analogy with the Einstein case are found to lie on the surface $Z_{0}=U_{0}=V_{0}=P_{0}=0$, with $P$ given by (23). We are interested in regular black hole solutions. A regular horizon is present if $R^{2} \equiv e^{2 \zeta-\eta-\chi}$ is finite at the critical points. Moreover, the dilaton is regular at the horizon if $e^{-2 \phi} \equiv e^{\eta-\chi}$ is finite there. These conditions are equivalent to the requirement that at the horizon $Z^{2} \sim U^{2} \sim V^{2}$. Since the critical points correspond to $\xi \rightarrow \pm \infty$, with $Z^{2} \sim e^{2 X_{0} \xi}, U^{2} \sim e^{2 Y_{0} \xi}, V^{2} \sim e^{2 W_{0} \xi}$, one obtains that the horizon is regular iff $X_{0}=Y_{0}=W_{0}=a$, for some real parameter $a$. To simplify the following discussion, we shall only consider points that satisfy this constraint.

The nature of the critical points can be investigated linearizing (8) and (14) around them. One obtains

$$
\delta Y^{\prime}=\delta X^{\prime}=\delta W^{\prime}=0, \quad \delta Z^{\prime}=a \delta Z, \quad \delta U^{\prime}=a \delta U, \quad \delta V^{\prime}=a \delta V,
$$

with eigenvalues $0(3), a(3)$. It results therefore that the critical point is an attractor for $a<0$ and a repellor for $a>0$.

The asymptotic behaviour of the solutions is dictated by the nature of the critical points at infinity $[8,1]$. These can be investigated using the variables $(24)$. The field equations at infinity $(t \rightarrow 0)$ read

$$
\begin{array}{lll}
\dot{t}=-f t, & \dot{y}=g-f y, & \dot{w}=h-f w, \\
\dot{z}=(1-f) z, & \dot{u}=(y-f) u & \dot{v}=(w-f) v,
\end{array}
$$

where a dot denotes $t d / d \xi, f=t^{2} F(1, y, w, z, u, v), g=t^{2} G(1, y, w, z, u, v), h=t^{2} H(1, y, w, z, u, v)$, and the variables are subject to the constraint

$$
2-y^{2}-w^{2}-2 z^{2}+2 q_{m}^{2} v^{2}+2 q_{e}^{2} u^{2}-\left(y^{2}-w^{2}\right)\left[4 z^{2}-3(y+w-2)^{2}\right] \frac{v^{2}}{z^{4}}=0 .
$$


The critical points at infinity are placed at $t_{0}=0$, with

a) $u_{0}=v_{0}=z_{0}=0, y=y_{0}, w=w_{0}$, with $y_{0}^{2}+w_{0}^{2}=2$.

b) $u_{0}=v_{0}=0, z_{0}=1, y_{0}=w_{0}=0$.

c) $v_{0}=0, u_{0}=1 / \sqrt{2 q_{e}^{2}}, z_{0}=1, y_{0}=1, w_{0}=0$.

e) $u_{0}=\sqrt{\frac{q_{m}^{2}+4}{\left(2 q_{m}^{2}+4\right) q_{e}^{2}}}, v_{0}=\frac{1}{\sqrt{2 q_{m}^{2}+4}}, z_{0}=1, y_{0}=w_{0}=1$.

The points a), b) and c) coincide with those obtained in the Einstein case, while the point e) is displaced due to the GB contribution. The most interesting feature is however that the critical point d) has disappeared. In particular, no solution with vanishing electric charge and unusual asymptotics exists in the GB case.

The eigenvalues of the linearized equations are

a) $0(3), 1, y_{0}, w_{0}$.

b) $-1(5),-2$.

c) $1,-1(3),-2(2)$.

e) $1,-1,-2(2),-\frac{1}{2}\left(1 \pm \sqrt{9+16 / q_{m}^{2}}\right)$.

They have the same sign as in the Einstein case, and hence analogous properties.

The resulting phase space portrait is rather similar to that of the Einstein system, except for the critical points d), that in the GB case are absent. In particular, all the trajectories corresponding to solutions with regular horizons start from the critical point at $Z_{0}=U_{0}=V_{0}=0, X_{0}=Y_{0}=W_{0}=a>0$ for a given value of the parameter $a$, and end at the points b), c) or e).

From the location of the critical points at infinity, one can deduce the asymptotic behaviour of the solutions $[9,1]$. The behaviour coincides in all cases with that found in the Einstein limit. In particular, in case b) solutions are asymptotically flat with asymptotically constant scalar field, while in case c) they behave like (20) and in case e) like (22). The existence of solutions with $a=0$ also suggests the existence of metrics with multiple horizons, i.e. with multiple roots of the metric functions, that should correspond to extremal black holes.

\section{Branch singularities}

In the previous section, we have studied the existence of solutions with regular horizons. Our method, however, does not give a complete characterization of the black holes. In particular, it does not permit to study the region inside the horizon and to ascertain the existence of inner horizons ${ }^{\dagger}$. Moreover, we are not able to relate the parameters $a, q_{m}$ and $q_{e}$ to the radius of the horizon. On the other hand, in GB models, branch singularities usually appear [10,7]: the requirement that they are shielded by an horizon imposes a minimal value for the horizon radius (and consequently for the mass of the black hole). In order to investigate the presence of these singularities, it is useful to study the behaviour of the fields near the outer horizon [11]. The calculation is most easily performed in coordinates of the form

$$
d s^{2}=-e^{\mu(R)} d t^{2}+e^{\lambda(R)} d R^{2}+R^{2} d \Omega^{2} .
$$

$\dagger$ Some results on this topic have been discussed using numerical methods in [7]. 
In this gauge, the field equations read

$$
\begin{aligned}
\phi^{\prime \prime}+ & \left(\frac{\mu^{\prime}-\lambda^{\prime}}{2}+\frac{2}{R}\right) \phi^{\prime}= \\
& -\frac{2 \alpha e^{-2 \phi}}{R^{2}}\left[\mu^{\prime} \lambda^{\prime} e^{-\lambda}+\left(1-e^{-\lambda}\right)\left(\mu^{\prime \prime}+\frac{\mu^{\prime}}{2}\left(\mu^{\prime}-\lambda^{\prime}\right)\right)\right]-\alpha\left(q_{m}^{2} e^{-2 \phi}-q_{e}^{2} e^{2 \phi}\right) \frac{e^{\lambda}}{R^{4}} \\
\frac{\lambda^{\prime}}{R}- & \frac{1-e^{\lambda}}{R^{2}}=\phi^{2}+\frac{4 \alpha}{R^{2}} e^{-2 \phi}\left[\left(1-3 e^{-\lambda}\right) \mu^{\prime} \phi^{\prime}-2\left(1-e^{-\lambda}\right)\left(\phi^{\prime \prime}-2 \phi^{2}\right)\right]+\alpha\left(q_{m}^{2} e^{-2 \phi}+q_{e}^{2} e^{2 \phi}\right) \frac{e^{\lambda}}{R^{4}} \\
\frac{\mu^{\prime}}{R}+ & \frac{1-e^{\lambda}}{R^{2}}=\phi^{\prime 2}+\frac{4 \alpha}{R^{2}} e^{-2 \phi}\left(1-3 e^{-\lambda}\right) \mu^{\prime} \phi^{\prime}-\alpha\left(q_{m}^{2} e^{-2 \phi}+q_{e}^{2} e^{2 \phi}\right) \frac{e^{\lambda}}{R^{4}} \\
\mu^{\prime \prime}+ & \left(\frac{\mu^{\prime}}{2}+\frac{1}{R}\right)\left(\mu^{\prime}-\lambda^{\prime}\right)= \\
& -2 \phi^{\prime 2}-\frac{8 \alpha e^{-2 \phi}}{R} e^{-\lambda}\left[\left(\mu^{\prime \prime}+\frac{\mu^{\prime}}{2}\left(\mu^{\prime}-3 \lambda^{\prime}\right)\right) \phi^{\prime}+\mu^{\prime}\left(\phi^{\prime \prime}-2 \phi^{\prime 2}\right)\right]+2 \alpha\left(q_{m}^{2} e^{-2 \phi}+q_{e}^{2} e^{2 \phi}\right) \frac{e^{\lambda}}{R^{4}}
\end{aligned}
$$

In order to find an analytical expression for the minimal value of the radius, we assume that near the horizon $R=R_{h}$ the fields behave as

$$
\begin{gathered}
e^{-\lambda}=\Lambda\left(R-R_{h}\right)+\ldots, \quad e^{\mu}=\mathrm{M}\left(R-R_{h}\right)+\ldots, \\
\phi=\phi_{h}+\phi_{h}^{\prime}\left(R-R_{h}\right)+\ldots,
\end{gathered}
$$

for some constants $\Lambda, \mathrm{M}, \phi_{h}$ and $\phi_{h}^{\prime}$. Let us first consider the case $q_{e}=0$. Substituting into the field equations, one obtains from (28)

$$
\Lambda=\frac{1}{R_{h}} \frac{1-g_{m}}{1-f}
$$

where

$$
g_{m}=\frac{\alpha q_{m}^{2} e^{-2 \phi_{h}}}{R_{h}^{2}}, \quad f=\frac{4 \alpha \phi_{h}^{\prime} e^{-2 \phi_{h}}}{R_{h}} .
$$

Moreover, combining (26) and (29), it results that

$$
f=\frac{8 \alpha^{2} e^{-4 \phi_{h}}}{R_{h}^{3}}\left[\Lambda+\frac{2}{R_{h}}\left(1+\Lambda R_{h} f-\frac{g_{m}}{\Lambda R_{h}}\right)\right]-\frac{4 \alpha e^{-2 \phi_{h}} g_{m}}{\Lambda R_{h}^{3}}
$$

and hence, substituting (30) in (32), after some algebraic manipulations one obtains a second degree algebraic equation for $f$ :

$$
\left[1-\left(1+\bar{\alpha}+\bar{\alpha}^{2}\right) g_{m}\right] f^{2}-\left[1-\left(1+2 \bar{\alpha}+\bar{\alpha}^{2}\right) g_{m}+\bar{\alpha}^{2} g_{m}^{2}\right] f+\frac{1}{2}\left[3 \bar{\alpha}^{2}-2 \bar{\alpha}(1+3 \bar{\alpha}) g_{m}+\bar{\alpha}^{2} g_{m}^{2}\right]=0,
$$

where we have defined $\bar{\alpha}=4 \alpha e^{-2 \phi_{h}} / R_{h}^{2}$.

In order to have real solutions for $f$, the discriminant $\Delta$ of the equation must be nonnegative, i.e.

$$
\Delta=\left(1-g_{m}\right)^{2}\left[1-6 \bar{\alpha}^{2}+2 \bar{\alpha}^{2}\left(1+3 \bar{\alpha}+3 \bar{\alpha}^{2}\right) g_{m}+\bar{\alpha}^{4} g_{m}^{2}\right] \geq 0 .
$$

The inequality holds for any $g_{m}$ as long as $\bar{\alpha}^{2} \leq 1 / 6$, i.e. $R_{h}^{2} \geq \alpha e^{-2 \phi_{h}} / 4 \sqrt{6}$. The same restriction holds also for neutral solutions [11,7]. However, in the charged case (34) can be satisfied also when $\bar{\alpha}^{2} \geq 1 / 6$, if

$$
g_{m} \geq \frac{-\left(2+2 \bar{\alpha}+3 \bar{\alpha}^{2}\right)+\sqrt{3\left(1+4 \bar{\alpha}+9 \bar{\alpha}^{2}+6 \bar{\alpha}^{3}+3 \bar{\alpha}^{4}\right)}}{\bar{\alpha}^{2}} .
$$


The same result was also obtained in [8] for SU(2) Yang-Mills monopoles. It is also interesting to remark that the vanishing of the prefactor in $(34), g_{m}=1$, corresponds to extremal black holes.

When $q_{e} \neq 0$, one obtains similar results. In this case, defining $g_{e}=\alpha e^{2 \phi_{h}} q_{e}^{2} / R_{h}^{2}$,

$$
\Delta=\left(1-g_{m}-g_{e}\right)^{2}\left[1-6 \bar{\alpha}^{2}+2 \bar{\alpha}^{2}\left(1+3 \bar{\alpha}^{2}\right)\left(g_{m}+g_{e}\right)+\bar{\alpha}^{4}\left(g_{m}+g_{e}\right)^{2}+6\left(g_{m}-g_{e}\right)\right],
$$

which is nonnegative for $\bar{\alpha}^{2} \leq 1 / 6$. If instead $\bar{\alpha}^{2} \geq 1 / 6$ a complicated constraint must be obeyed by the charges. Again, the case $g_{m}+g_{e}=1$ corresponds to the extremal limit.

\section{Conclusions}

The study of charged solutions of the dilatonic Einstein-GB model has revealed the presence of some difference from the pure Einstein case. In particular, non-asymptotically flat solutions with vanishing electric charge are absent in the GB case. The conditions for the absence of naked branch singularity is identical to that of the neutral case, but the possibility exists of solutions violating this condition, if the charges satisfy some inequalities. Also interesting is the existence of extremal solutions, which were not considered in previous investigations.

\section{References}

[1] M. Melis and S. Mignemi, Class. Quantum Grav. 22, 3169 (2005).

[2] B. Zwiebach, Phys. Lett. B156, 315 (1985); B. Zumino, Phys. Rep. 137, 109 (1986); D.J. Gross and J.H. Sloan, Nucl. Phys. B291, 41 (1987).

[3] K.C.K. Chan, J.H. Horne and R. Mann, Nucl. Phys. B 447, 441 (1995);

[4] G.W. Gibbons and K. Maeda, Nucl. Phys. B298, 741 (1988).

[5] D. Garfinkle, G.T. Horowitz and A. Strominger, Phys. Rev. D43, 3140 (1991).

[6] S. Mignemi and N.R. Stewart, Phys. Rev. D47, 5259 (1993); S. Mignemi, Phys. Rev. D51, 934 (1995).

[7] T. Torii, H. Yajima, and K. Maeda, Phys. Rev. D55, 739 (1997); S.O. Alexeyev and M.V. Pomazanov, gr-qc/9706066.

[8] P. Kanti and K. Tamvakis, Phys. Lett. B392, 30 (1997).

[9] S. Mignemi and D.L. Wiltshire, Class. Quantum Grav. 6, 987 (1989); D.L. Wiltshire, Phys. Rev. D44, 1100 (1991); S. Mignemi and D.L. Wiltshire, Phys. Rev. D46, 1475 (1992); S.J. Poletti and D.L. Wiltshire, Phys. Rev. D50, 7260 (1994); S. Mignemi, Phys. Rev. D62, 024014 (2000).

[10] D.G. Boulware and S. Deser, Phys. Rev. Lett. 55, 2656 (1985); D.L. Wiltshire, Phys. Lett. B169, 36 (1986).

[11] P. Kanti, N.E. Mavromatos, J. Rizos, K. Tamvakis and E. Winstanley, Phys. Rev. D54, 5049 (1996).

[12] A. Shapere, S. Trivedi and F. Wilczek, Mod. Phys. Lett. A6, 2677 (1991). 